\title{
4.3 AN ANALYSIS OF RISK KNOWLEDGE CREATION FOR EFFECTIVE MANAGEMENT AND LEADERSHIP - CASE STUDIES OF JAPANESE COMPANIES
}

\begin{abstract}
Summary: Our research discusses risk knowledge creation based on the knowledge creation model introduced by Nonaka and Takeuchi (1995). As in their model, the risk knowledge creation model we propose in this paper consists of four conversion modes: socialization, externalization, combination, and internalization. The purpose of this research is to show the essential factors for effective creation of risk knowledge. We use a case study method to achieve this. The case study we conducted revealed that giving too much weight to socialization is dangerous. That it is important for externalization to analyze common features or factors of many phenomena or incidents. That justifying conducting preventative measures of risk in combination is critical. Finally, that motivating people to keep their tacit risk knowledge is significant for internalization. As a consequence, we concluded that effective risk knowledge creation requires leadership that fosters a culture in which risk is discussed.
\end{abstract}

Keywords: risk, explicit and tacit knowledge, knowledge creation, leadership

\section{INTRODUCTION}

Companies must take certain risks to improve profits, yet a company's survival can be threatened by excessive risk taking. Therefore, companies should manage serious risks appropriately. However, risk taking is more complex today than ever before. Under the present circumstances, it is commonly assumed that companies take too much risk by overlooking critical variables, underestimating serious risks, and reacting inadequately to risks.

Companies need to regularly review whether they have overlooked risks that may prove important in the future, what kind of impact current risk taking has on business, and how to respond to risks more effectively. In order to achieve these objectives it is necessary for the company to not only ensure that its existing risk knowledge is adequate, but also to continually update and renew its knowledge of risk. That is to say, "risk knowledge creation" is required in modern business. Based on this perspective, it is essential for modern management research to study risk knowledge creation.

Existing studies have already highlighted the importance of risk knowledge. COSO (2004) indicated the importance of the Enterprise Risk Management (ERM) framework, which consists of eight interrelated components. In this framework, risk knowledge (capability, skill, and information) can be positioned in the "internal environment" or "information and communication" section. Although existing risk management research has discussed risk knowledge itself in this manner, few studies have focused on risk knowledge creation.

Some studies have already incorporated the concept of risk as it relates to knowledge or knowledge creation research. For example, Cooper (2003) examined risk in innovation during product development, and Kan and Tsai (2004) analyzed knowledge of health risks. Admittedly, this research was conducted on risk and knowledge or knowledge creation. However, little is actually known about risk knowledge creation, namely the manner in which risk knowledge is created and the factors necessary to promote it.

In order to clarify this subject, we will analyze both the areas of risk management and knowledge creation. In Chapter 2, we will review the definitions of several key concepts as 
well as a theory on knowledge creation. In Chapter 3, we will present a framework based on these concepts and this theory. In Chapter 4, we will use case studies to show the essential factors for effective creation of risk knowledge. Finally, in Chapter 5, we will reveal the implications that have become apparent from the above analysis and offer further direction for this area of study.

\section{REVIEWING KEY CONCEPTS}

\subsection{RISK AND KNOWLEDGE}

We define risk as the possibility of any adverse impact on the company. Knight (1921) presented a well-known definition of risk, defining it as uncertainty that can be measured in some way. While uncertainty that is difficult to calculate using a quantitative approach was "true uncertainty". It was presupposed by Knight (1921) that a company gains profits by taking on true uncertainty. However, we do not limit the concept of risk to what can be understood based on occurrence probability measured quantitatively. This is because, whether or not the probability and the potential loss can be calculated by a certain technique, if there is a possibility of any adverse impact on business or profits a company must manage it.

Nonaka and Takeuchi (1995) defined knowledge as "justified true belief" and pointed out that this belief arose from information. Based on the above statement, we define knowledge as information used in decision-making, actions and the belief formed by the information. This definition includes quantitative information, as well as qualitative (meanings, perspectives, principles, and so forth). Knowledge can be divided into explicit knowledge and tacit knowledge. The former is objective knowledge and is easy to transfer because it can easily be expressed in language or a numerical form. The latter is subjective knowledge. Tacit knowledge has the cognitive aspects, such as a mental model and the technical aspects, such as know-how. Whatever the case may be, tacit knowledge is difficult to pass on to others.

\subsection{KNOWLEDGE CREATION}

According to Nonaka and Takeuchi (1995), knowledge creation is conducted through four conversion modes: socialization, externalization, combination, and internalization (Figure1).

First, socialization creates tacit knowledge from other tacit knowledge through the act of sharing experiences without using language or numbers. The typical example of socialization is OJT (On the Job Training). OJT is carried out to acquire tacit knowledge, such as the knowhow of work by observing practices and imitation.

Second, externalization verbalizes tacit knowledge into a clear concept. This is a significant process, since an ambiguous matter will be transferred to a certain analogy, hypothesis, model or similar state. Nonaka and Takeuchi (1995) introduce the example of Matsushita Denki Co.,Ltd. (Now Panasonic. Henceforth, Matsushita Denki), developing a "home-bakery", which is an automatic bread-baking machine for home use. The idea of more delicious bread was expressed as "Hotel bread", and the techniques of experienced bakers were showed as the concept of "twisting stretch" for example.

Third, combination organizes explicit knowledge, including categorizing and sharing of it. In addition, it is important to link middle-range concepts with grand concepts. This means that a concrete concept, such as a product concept, is connected with integrative concepts, such as the vision and mission of the company. For example, we could present the practicing sales promotion by using a POS (Point of Sales) system. With a POS system, we could know about the quantity of purchased products and the areas where they were bought. With POS data, we 
could learn consumers' trends and thus devise new methods of sales promotion. It creates explicit knowledge from explicit knowledge, so it could be included in combination.

Finally, internalization is the process of changing explicit knowledge into tacit knowledge and gaining tacit knowledge, such as a new mental model or know-how through learning by doing. For example, Nonaka and Takeuchi (1995) presented the case of Matsushita Denki's reduction of working hours. To improve creativity, Matsushita Denki notified people of a reduction in working hours. However, the implementation was not made clear. So they experimentally shortened working hours to 150 hours a month. With this method, workers could get used to working within the shortened time and how to cope with their work instinctively. Such "learning by practice" is relevant to internalization.

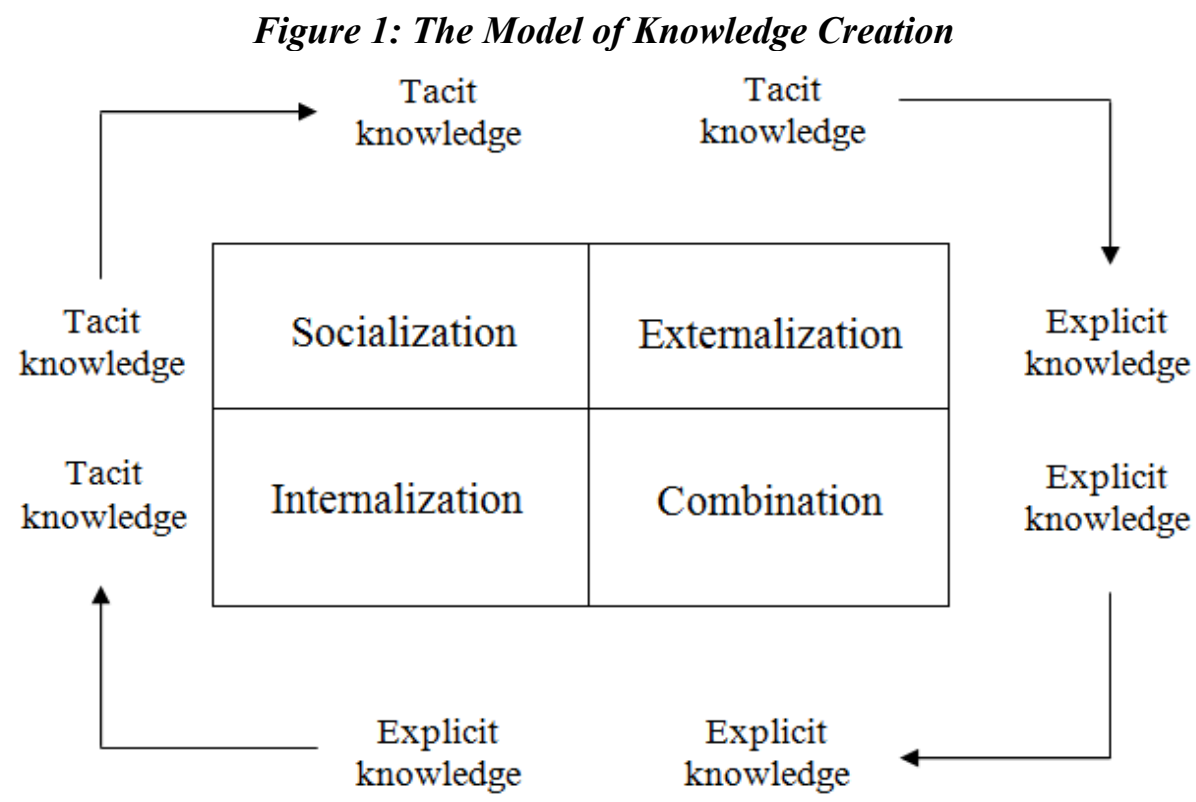

Source: Nonaka and Takeuchi (1995) Figure3-2.

If tacit knowledge acquired in the internalization mode is shared with other members of the organization, then it can be socialization. By using the above mentioned examples, new members could adapt to working in a shorter time than before by imitating on OJT. In this way, knowledge creation is practiced by repeating the spiral through four processes that are related to explicit and tacit knowledge.

\section{RISK KNOWLEDGE CREATION}

\subsection{EXPLICIT RISK KNOWLEDGE AND TACIT RISK KNOWLEDGE}

We can divide risk knowledge into explicit risk knowledge and tacit risk knowledge. Explicit risk knowledge is knowledge, which can be expressed clearly in words, language or figures for addressing risk. For example, a numeric statement of accounts or behaviour standards that describe knowledge necessary for understanding and dealing with risk, such as staff and office regulations, instruction manuals and so forth. Calculated numerical values by such quantitative methods of VaR (Value at Risk) or EaR (Earnings at Risk) also fit in here. When a company has such explicit knowledge and it is ready for use, the company can confirm the risks that are in place, what to pay attention to, and where to place restrictions.

Tacit risk knowledge is difficult to express in language or as a numerical value. It contains things such as a company's attitude to risks, ways of understanding risk, non-numeric evaluation of risk and know-how relating to non-verbal responses. 
A company's attitude to risks and the ways of understanding risk relates to perceptual differences in the risk depending on one's experience or situation. For example, even if people in a company observe the same product, members of the production department would examine the risk of the products' design and assembly, whereas members of the sales department would examine the risk relating to customers and the sales method. This might be tacit knowledge because the person themselves does not explicitly know the framework of the risk or mental model.

Non-numeric evaluation of risk means to evaluate risk without a numeric method. Usually, a risk evaluation is conducted using a numeric method e.g., about $25 \%$, three times a decade, loss of 100 thousand yen and so on. Whereas someone with abundant experience and technical knowledge might recognize a risk that would cause a loss but the probability or scale of it could not be expressed explicitly. For example, one might feel somewhat unsure about how to speak and behave around the person in charge. It is difficult to explain this to other people, and is not an objective evaluation. Not all kinds of risk can be numerically evaluated, so we could say this is one side of risk evaluation.

Know-how relating to non-verbal responses is a measure of risk that is difficult to convey to other people. For example, inspections carried out by someone who has long-term experience and special skills or preventing reduced motivation by personal charm or skilful speaking. Even if these responses to the risks are dealing with them effectively, it is difficult to express them numerically or document it. Therefore, this is an area of the tacit risk knowledge.

\subsection{THE PROCESS OF RISK KNOWLEDGE CREATION}

We will examine risk knowledge creation using the theory developed by Nonaka and Takeuchi (1995). Figure 2 is the risk knowledge creation model with the added concept of risk to Figure 1. Based on this model, we will consider each four conversion modes. As mentioned at the introduction, the four modes of our model are similar to those of Nonaka and Takeuchi's (1995) model. However, knowledge which is related to these modes is not explicit knowledge and tacit knowledge in the case of risk knowledge creation but explicit risk knowledge and tacit risk knowledge.

Figure 2: The Model of Risk Knowledge Creation

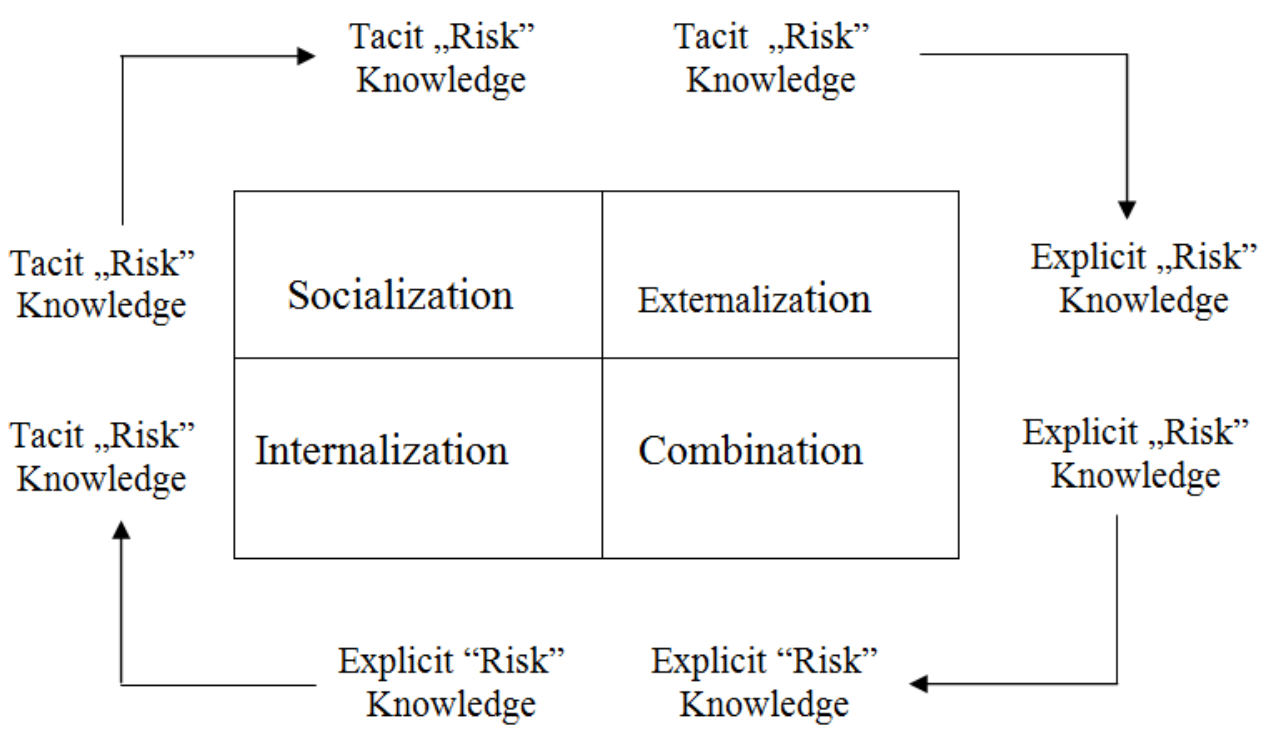

Source: based on Figure 1 
Socialization of risk knowledge means to share and learn tacit risk knowledge such as ways of understanding risk, attitudes to risk, and operations know-how regarding avoiding accidents. For example, analyzing cases of failure that occurred in other companies or arguing about possible accidents on any committees related to risk management are socialization. In the activity, some people who have various specializations join together and debate the risk from various viewpoints beyond ones' specialization. In this regard, members should share and modify frameworks or mental models of how to understand risks, through the experience of different ways of understanding of risks or attitudes of addressing risks.

Externalization of risk knowledge makes important tacit risk knowledge into explicit risk knowledge, and thus states it clearly so it can be understood by other people. For example, pointing out events where a risk was not recognized or highlighted in any manuals would be externalization. When nobody except the person who raised the issue recognizes the risk, it is especially critical that externalization of risk knowledge occurs. As since explicit knowledge is easy to transmit to other people those who do not have tacit knowledge can understand new risks through that externalization.

Combination of risk knowledge means to convey and diffuse explicit risk knowledge, as well as creating and systemizing new explicit risk knowledge from it. Furthermore, the importance of relating medium range and grand concepts, as described in Chapter 2.2, is one aspect of combination. It relates individual concepts, which were externalized explicit risk knowledge, and overall concepts, such as management principles or strategies. For example, to update the behaviour standards or to make new manuals by considering accident reports or risk information analyzed by specialists would be combination. Moreover, to convey these new standards or manuals to the members who had not acquired this new explicit knowledge yet would be combination, since notification or diffusion is part of it.

Internalization of risk knowledge means to act based on explicit risk knowledge and then create tacit risk knowledge. We could say it is internalization of risk knowledge to recognize risk and respond to it appropriately without referencing any manual or examples. In addition, to construct a new risk mental model or accumulate corresponding know-how by adapting explicit risk knowledge to other fields or matters is also internalization of risk knowledge.

\section{CASE STUDIES}

Now, we will analyze how and to what we should pay attention to execute these four processes of risk knowledge creation effectively, illustrated with related examples.

\subsection{SOCIALIZATION}

Firstly, we should point out the risk that knowledge creation gives too much weight to socialization. Certainly, it is important to share tacit risk knowledge because not all the tacit knowledge can become explicit. However, if people rely excessively on socialization, dangerous methods can be inherited or those who have erroneous beliefs might appear and increase.

In 1999, in Tokai village, Ibaragi prefecture in Japan, JCO Inc. (henceforth JCO) had a criticality accident, a type of nuclear incident, in which two people died and more than 600 people were affected by radiation. In this accident, workers at the job site were not educated adequately and had little knowledge of uranium or criticality (Nanasawa, 2005). This became a key issue. The knowledge necessary for operations and management did not depend solely on education based explicit knowledge, but also greatly on tacit knowledge. If such circumstances where knowledge is not effectively shared, last for a long period of time it is to be expected that a dangerous accident or mistake could occur, as the workers could not 
recognize dangers. Furthermore it might be difficult to suggest necessary modifications or corrections for operations, as they had not conveyed explicit knowledge such as background information on operations or management.

\subsection{EXTERNALIZATION}

Second, we will examine externalization (This case was written based on an interview with Ryosuke Hirai, senior adviser in the CSR Office, RICOH Inc., December 21, 2005). In this paper, we will point out the importance of externalizing vital risks for the firm by analyzing common features or factors of specific phenomena or incidents. An important example to examine is that of RICOH Inc. (henceforth RICOH). In late 1990, RICOH examined various accidents or mistakes which had occurred. They discovered two issues.

The first finding was that many problems had arisen at affiliated companies, yet the parent company assumed responsibility for these problems. Currently RICOH executes risk management as a whole company, but at that time it was only in the main part of the parent company. Therefore, the department of general affairs, which supervised risk management, suggested at a management meeting that it should be necessary for the whole RICOH group to execute risk management. Risk management for a whole company is called ERM (Enterprise Risk Management), and has been examined in many countries since 2000. COSO (2004), which we introduced in chapter 1, is an integrated ERM framework. In late 1990, RICOH could recognize the importance of ERM ahead of the rest of the world.

The second finding was the revelation of patterns of fraud. As a result of the legal department's examination of examples, it became clear that many revelations of patterns of fraud are due to whistle-blowing activities. Therefore the legal department suggested at a management meeting that it should be necessary to build an internal reporting system. That is, the department pointed out that RICOH needed to build a system that could quickly accept risk reports and respond to risk. This system enables a company to recognize problems before the risk becomes reality or at an early stage of incidence.

\subsection{COMBINATION}

In regards to combination, we can point out the importance of justifying the response to risks. This is because even tackling mistakes or accidents beforehand generates costs such as coordination within the organization or payment of insurance fees. However it is hard to understand the consequences of actions like this directly or in the short term. For example, the ideal result of preventing mistakes is that "nothing" occurs. Therefore, it is not easy to understand the direct relationship between tackling issues beforehand and the results. In other words, initiatives to cope with risk might be given lower priority than actions, which make profit such as $R \& D$, production or sales. Thus, with combination, how to justify preventative measures towards risk becomes an important issue. Below, we examine and compare the cases of Fuji-Xerox Co. Ltd, (henceforth Fuji Xerox) and Sanyo Denki Co. Ltd (henceforth Sanyo Denki).

Fuji-Xerox's company policy takes a serious view on employees' health and safety. Since the first half of the 1990s, they have put in place countermeasures in case of earthquakes. So during the Niigata-Chuetsu Earthquake in 2004, Fuji-Xerox could quickly return to normal conditions while many other companies had to cease operations. On the other hand, a semiconductor factory of Sanyo Denki's subsidiary completely collapsed, and they suffered a loss of 50 billion yen. Sanyo Denki had put off taking out earthquake insurance on the subsidiary as it was concerned over the possibility of not receiving full coverage or compensation. However it is conventional wisdom in the industry to take out insurance 
because the production facilities for semiconductors are so expensive (Nihon Keizai Shinbun, December 24, 2004).

What we have to comment on here is why Fuji-Xerox was willing to prepare for earthquakes to its subsidiary and could take detailed measures in advance. A mid-range concept, such as a specific matter on earthquake countermeasures is justified by a perspective from a grand concept, such as a mission statement or corporate strategy. In such circumstances, the cost for coordination or preparation is estimated and viewed as an inevitable cost, so it is easy to progress with arrangements beforehand. Whereas for SanyoDenki, insurance costs were not warranted, because of the limiting of the compensation range. So we can conclude that for promoting combination of risk knowledge creation, it is important to justify individual actions needed to address risks (a mid-range concept) from the viewpoint of a mission or strategy of a company(a grand concept).

\subsection{INTERNALIZATION}

Forth, as we have clarified in Chapter 2.2, it is necessary to act or learn for internalization. However, any risk no matter how small it is, can cause problems for a firm, such as accidents or mistakes. Therefore, we can say that simulated experiences of accidents or mistakes are necessary. A fire evacuation drill being a typical example. However, activities or training exercises like this can turn into a mere formality. They can become a mere ceremony to confirm explicit knowledge. Furthermore, as time goes by, obtained tacit knowledge or feelings (also tacit) might be lost. To prevent it merely being a formality or loosing tacit knowledge, and thus to be able to internalize knowledge effectively, actions should be taken. For example, NEC (Nihon Electric Company) introduces two cases per month in their mail magazine that draw attention to risk issues (Nihon denki, 2004). By introducing a variety of field cases and stimulating interest in people they can prevent simulated experiences turning into a mere formality, control the loss of tacit knowledge and keep people aware of risk, not with huge and low frequency actions but small and high frequency.

If such measures are continued, it is possible that people in the company can recognize a sense of incongruity or risks from various phenomena. If these customs and techniques are shared by the company (socialization), it leads to conceptualizing new risk (externalization) and updating the behaviour standards (combination). Such a company can respond to many risks, since people recognize and try to cope with risks before they become reality.

\section{CONCLUSIONS}

In this paper we have indicated that studies on risk management and knowledge creation rarely pay direct attention to risk knowledge creation. We have examined what risk knowledge is, how we can understand risk knowledge creation using case studies and the theory of Nonaka and Takeuchi (1995), and what to focus on to make these practices effective. As for socialization, we have indicated that if socialization of risk knowledge creation is over stressed, the possibility of risk occurrence, in the form of accidents or mistakes, might increase. As for externalization, we have studied the necessity of expressing risk, which could be serious, in the company's own context. As for combination, it is difficult to understand risk and the results of preventative measures in the short-term or directly. So we have pointed out the importance of justifying preventative measures by positioning them as a company-wide issue. As for internalization, we have shown that it is necessary to stimulate interest in the subject through education or learning and to have methods to maintain gained tacit knowledge. In short, we have suggested that learning and experiences should be on a small scale and high frequency incorporated into daily operations, not as special activities. 
Now, we will present some implications and contributions, which could be of importance to the leadership. When a company finds unrecognized risk, it is essential that someone points it out, discussions are held and measures created, yet passive leaders might possibly suppress these actions. If this attitude is normalized, then recognized serious risks could be ignored without being addressed as an issue. So it is necessary for leaders of organizations to actively point out possible risk in order to facilitate risk knowledge creation and to be open to discussion about risk with subordinates. A leader's most important role is to foster a culture in which risk is discussed.

Moreover, it is important to document risk and assumed crisis for practical applications of risk knowledge creation. Companies may be negative about enforcing it, since it requires costs and highlights threats and weak points of the company. However, starting documentation of risks or crises is optimal for practical applications of risk knowledge creation. As in order to document risk and crises, various people in the company must argue, conceptualize and categorize them. The series of these processes includes socialization, externalization and combination which are critical for risk knowledge creation.

Lastly, we will suggest a future research topic. In chapter 4, we analyzed each of the four processes by illustrating specific cases. From these analyses, we have clarified some crucial issues in each process. However we have not analyzed the connection sequence of the four processes. Henceforth, we could say that an analysis describing the four processes in detail would be necessary to understand the dynamic aspect of risk knowledge creation.

\section{REFERENCES}

1. Cooper, L. (2003) "A research agenda to reduce risk in new product development through knowledge management: a practitioner perspective", Journal of Engineering \& Technology Management, Vol.20 (1-2) , pp.117-140.

2. COSO (Committee of Sponsoring Organizations of the Treadway Commission) (2004) Enterprise Risk Management - Integrated Framework.

3. Kan, K and W. Tsai (2004) "Obesity and Risk Knowledge", Journal of Health Economics, Vol.23 (5) , pp.907-934.

4. Knight, F. H. (1921) Risk, uncertainty and profit, Boston: Houghton Mifflin.

5. Nonaka, I and H. Takeuchi (1995) The knowledge-creating company: how Japanese companies create the dynamics of innovation, New York: Oxford University Press.

6. Nanasawa, K. (2005) Tokaimura rinkaijiko heno michi: Haraware nakatta anzen kosuto (The whole circumstances of the criticality accident in Tokai village: The unpaid safety costs), Tokyo:Iwanami shoten(in Japanese).

7. Nihon denki (2004) Jissenteki CSR keiei no susumekata (How to develop practical CSR management.), Tokyo: Nikkagiren (in Japanese).

8. Nihon Keizai Shinbun (Japanese economic newspaper), December 24, 2004, p.1 (in Japanese). 